\title{
Molecular Defect of Spectrin in
}

\section{Hereditary Pyropoikilocytosis}

\author{
ALTERATIONS IN THE TRYPSIN-RESISTANT DOMAIN \\ INVOLVED IN SPECTRIN SELF-ASSOCIATION
}

\author{
Jack Lawler, Shih-Chun Liu, and Jiri Palek, Departments of Research and \\ Medicine, St. Elizabeth's Hospital of Boston and Tufts University School of \\ Medicine, Boston, Massachusetts 02135 \\ Josef Prchal, Department of Medicine, University of Alabama Medical School, \\ Birmingham, Alabama 35294
}

\begin{abstract}
A B S T R A C T In hereditary pyropoikilocytosis (HPP) the erythrocyte membrane skeleton exhibits mechanical instability that can be correlated to defective selfassociation of spectrin heterodimers. To detect structural changes in the functional domains of HPP spectrin we have examined the peptide pattern produced by limited tryptic digestion of spectrin extracts from two families that contain three HPP patients. Limited tryptic digestion of all three HPP patients revealed a similar and reproducible decrease in the staining intensity of an 80,000-, and 22,000-, and an 88,000-dalton polypeptide with a concomitant increase in a 74,000 - and a 90,000 -dalton polypeptide as compared with controls. Only changes in the $80,000-$, and 74,000-, and 22,000-dalton polypeptides could be correlated to defective spectrin self-association and the amount of spectrin dimers in $0^{\circ} \mathrm{C}$ extracts of the HPP patients and their affected kindred. Similar results were obtained when the tryptic digests were analyzed by two-dimensional isoelectric focusing/sodium dodecyl sulfate-polyacrylamide gel electrophoresis with the affected 74,000and 80,000-dalton polypeptides focusing into multiple spots ranging in isoelectric point from 5.3-5.4. When HPP spectrin dimers and tetramers were separated and subjected to trypsin digestion, changes in the 80,000 -, 74,000 -, and 22,000 -dalton polypeptides were found predominantly in the spectrin dimer pool. Similar results were obtained for spectrin from two of the probands' mother, whom we have identified as an HPP carrier. We
\end{abstract}

This work was presented, in part, at the American Federation for Clinical Research, Wash., DC, 9 May 1982.

Received for publication 1 March 1982 and in revised form 25 June 1982. conclude that these HPP patients contain a population of normal, (principally tetrameric) and mutant (principally dimeric) spectrin. The latter is characterized by a defective spectrin dimer self-association due to conformational changes that affect the 80,000 -dalton domain.

\section{INTRODUCTION}

Normal erythrocyte morphology and structural integrity is maintained, in part, by a submembrane skeleton comprised primarily of spectrin, actin, and polypeptide $4.1(1-5)$. As recently reviewed, the prevailing evidence suggests that the major structural subunits of the skeleton are heterodimers of spectrin $\alpha$ - and $\beta$-chains, which by head-to-head self-association form tetramers and, possibly, higher oligomers (1-5). The tetramers, in turn, are thought to be assembled into a two-dimensional network via their interaction with band 4.1, which binds to the distal end of the heterodimers and facilitates their binding to actin oligomers.

Under conditions that thermodynamically favor preservation of the native species, spectrin is extracted from normal erythrocyte ghosts as tetramers and highmolecular weight complex with only a small percentage $(5 \pm 2 \%)$ in the heterodimer state (4-6). In contrast, in the erythrocytes from patients with hereditary pyropoikilocytosis (HPP) ${ }^{1}$ and some forms of hereditary elliptocytosis (HE) the percentage of spectrin in the

'Abbreviations used in this paper: DFP, diipopropyl fluorophosphate; DTT, dithiothreitol; HE, hereditary elliptocytosis; HPP, hereditary pyropoikilocytosis; SDS-PAGE, sodium dodecyl sulfate polyacrylamide gel electrophoresis; TPCK, L-(tosylamido-2-phenyl) ethyl chloromethyl ketone. 
heterodimer state increases to $30-38 \%$ and $15-30 \%$, respectively (7-9). The increased level of heterodimers correlates well with the mechanical instability of the HPP membrane skeletons, presumably owing to the reduced ability of spectrin to cross-link other skeletal components.

Limited tryptic digestion of spectrin has been used to identify some of the functional domains of spectrin $(10,11)$. An 80,000-dalton terminal portion of the $\alpha$ chain has been reported to contain the domain involved in tetramer formation (11-13). Further tryptic cleavage of the 80,000 -dalton fragment to 74,000 daltons destroys the ability of this fragment to bind to native spectrin, suggesting that a terminal 6,000-dalton portion is essential for preservation of function (13). Recently, an atyptical tryptic peptide pattern has been observed in one patient out of four studied with HE (14). Digestion of spectrin from patients with hereditary spherocytosis, with a variety of enzymes, reportedly produced normal peptide patterns $(14,15)$. In this paper we report on the peptide patterns produced by limited tryptic digestion of spectrin from two families with HPP.

\section{METHODS}

Clinical material. Three HPP patients from two unrelated Black families and their relatives were examined. Their clinical data fulfilling the criteria of HPP have been de- scribed $(8,16)$. A summary of the clinical and biochemical data is shown in Table I. Venous blood from these patients and their kindred was collected into sterile tubes containing citrate-phosphate-dextrose, or anticoagulant-citrate-dextrose, put on ice, and transported in insulated containers from Birmingham, $\mathrm{AL}$, to Boston, where it was kept at $4^{\circ} \mathrm{C}$ and analyzed no later than $3 \mathrm{~d}$. A control sample was sent along each time.

Spectrin extraction. Erythrocyte ghosts were prepared by the method of Dodge et al. (17). The ghosts were washed once in $0.1 \mathrm{mM} \mathrm{NaPO}$ (pH 8.0) and resuspended in an equal volume of $0.1 \mathrm{mM} \mathrm{NaPO}$ (pH 8.0), $0.1 \mathrm{mM}$ EDTA, and 0.1 $\mathrm{mM} \beta$-mercaptoethanol. Extracts that were subjected to column chromatography also contained $0.1 \mathrm{mM}$ phenylmethysulfonyl fluoride and $0.1 \mathrm{mM} N$ - $\alpha$ - $p$-tosyl-L-lysine chloremethyl ketone $\mathrm{HCl}$. After incubation at $0^{\circ} \mathrm{C}$ for $16 \mathrm{~h}$ or at $37^{\circ} \mathrm{C}$ for $20 \mathrm{~min}$ the samples were centrifuged at 250,000 $g$ for $35 \mathrm{~min}$, the supernatant was decanted and adjusted to a final concentration of $40 \mathrm{mM}$ Tris and $20 \mathrm{mM}$ sodium acetate buffer ( $\mathrm{pH}$ 7.4) containing $10 \mathrm{mM}$ dithiothreitol (DTT). These extracts were subjected to limited tryptic digestion or further fractionated by gel filtration chromàtography on a column $(2.1 \times 49 \mathrm{~cm})$ of Sepharose $4 \mathrm{~B}$ or density gradient centrifugation on linear $5-20 \%$ sucrose gradients for $15 \mathrm{~h}$ at $200,000 \mathrm{~g}$. Both separations were performed at $4^{\circ} \mathrm{C}$ in $10 \mathrm{mM} \mathrm{NaPO}$ ( $\left.\mathrm{pH} 7.4\right), 150 \mathrm{mM} \mathrm{NaCl}$, $5 \mathrm{mM} \beta$-mercaptoethanol, and $5 \mathrm{mM}$ EDTA.

Limited tryptic digestion. The protein concentration was determined with the Bio Rad protein assay kit (Bio-Rad Laboratories, Richmond, CA) using bovine serum albumin as standard. The spectrin extracts and purified fractions were adjusted to the same protein concentration before treatment with L-(tosylamido-2-phenyl)ethyl chloromethyl ketòne (TPCK) trypsin $(1: 10,1: 25,1: 50,1: 100,1: 200$, or 1:400 wt/

TABLE I

Summary of Clinical and Biochemical Data

\begin{tabular}{|c|c|c|c|c|c|c|c|c|}
\hline \multirow[b]{2}{*}{ Subject* } & \multirow[b]{2}{*}{ Age } & \multirow[b]{2}{*}{ Clinical presentation 1} & \multirow[b]{2}{*}{$\begin{array}{c}\text { Temperature } \\
\text { stability } \$\end{array}$} & \multirow[b]{2}{*}{$\begin{array}{l}\text { Stability of } \\
\text { triton shells" }\end{array}$} & \multirow[b]{2}{*}{$\begin{array}{c}\text { Sp-D in } \\
0^{\circ} \mathrm{C} \text { extracts }\end{array}$} & \multicolumn{3}{|c|}{ Tryptic fragments ${ }^{*}$} \\
\hline & & & & & & 80,000 & 74,000 & 22,000 \\
\hline & $y r$ & & & & $\%$ & & & \\
\hline \multicolumn{9}{|l|}{ Family E } \\
\hline Grandmother (G.E.) & $>50$ & $\mathbf{N}$ & $\mathbf{N}$ & $\mathbf{N}$ & $5 \pm 2$ & $\mathbf{N}$ & $\mathbf{N}$ & $\mathbf{N}$ \\
\hline Father (D.C.) & $>30$ & $\mathrm{~N}$, with $\mathrm{HbC}$ trait & $\mathbf{N}$ & $\mathbf{N}$ & $5 \pm 2$ & $\mathbf{N}$ & $\mathbf{N}$ & $\mathbf{N}$ \\
\hline Mother (C.E.) & $>30$ & $\mathrm{~N}$, with $\mathrm{HbC}$ trait & $\mathbf{N}$ & $\downarrow$ & $20 \pm 2$ & $\downarrow$ & $\uparrow$ & $\downarrow$ \\
\hline Sister (M.E.) & 12 & $\mathbf{N}$ & $\mathbf{N}$ & NA & $8 \pm 3$ & $\mathbf{N}$ & $\mathbf{N}$ & $\mathbf{N}$ \\
\hline Half-brother (J.E.) & 6 & $\mathbf{N}$ & $\mathbf{N}$ & NA & $10 \pm 3$ & $\mathbf{N}$ & + & $\mathbf{N}$ \\
\hline Proband (N.E.) & 14 & HPP, HbCC & $46^{\circ} \mathrm{C}$ & $\downarrow$ & $38 \pm 2$ & $\downarrow$ & $\uparrow \uparrow$ & $\downarrow$ \\
\hline \multicolumn{9}{|l|}{ Family B } \\
\hline Father (F.B.) & $>30$ & $\mathbf{N}$ & $\mathbf{N}$ & $\mathbf{N}$ & $5 \pm 2$ & $\mathbf{N}$ & $\mathbf{N}$ & $\mathbf{N}$ \\
\hline Mother (E.B.) & $>30$ & $\mathbf{N}$ & $\mathbf{N}$ & $\downarrow$ & $20 \pm 2$ & $\downarrow$ & $\uparrow$ & $\downarrow$ \\
\hline Proband (A.B.) & 1.5 & HPP & $45^{\circ} \mathrm{C}$ & $\downarrow$ & $30 \pm 4$ & $\downarrow$ & $\uparrow \uparrow$ & $山$ \\
\hline Proband (D.B.) & 4 & HPP & $45^{\circ} \mathrm{C}$ & $\downarrow$ & $34 \pm 4$ & $山$ & $\uparrow \uparrow$ & $\downarrow$ \\
\hline
\end{tabular}

- All of the subjects in this study are Black.

I , normal.

Temperature at which abnormal erythrocytes fragment and form membrane projections.

"From reference 8 . The mechanical stability of triton shells was classified as normal $(\mathrm{N})$, slightly decreased ( $(\mathrm{l})$, or considerably decreased (山). NA, data not available.

II From reference 8.

$\cdots$ The quantity of affected peptides was classified as normal $(N)$, slightly increased $(+)$, increased $(\uparrow)$, decreased $(l)$, considerably increased $(\pi)$, or considerably decreased $(\downarrow)$. 
wt) for $20 \mathrm{~h}$ at $0^{\circ} \mathrm{C}$. Digestion was terminated by addition of $1 \mathrm{mM}$ diisopropyl fluorophosphate (DFP) or by heating the samples to $100^{\circ} \mathrm{C}$ for $1 \mathrm{~min}$ in the presence of $1 \%$ sodium dodecyl sulfate (SDS) and $20 \mathrm{mM}$ DTT.

Protein electrophoresis. Digests were electrophoresed on discontinuous SDS-polyacrylamide tube or slab gels by the procedure of Laemmli (18). The stacking gel was comprised of $2.67 \%$ acrylamide and $0.10 \%$ bisacrylamide and the separating gel was comprised of $10 \%$ acrylamide and $0.38 \%$ bisacrylamide. The gels were stained for protein with Coomassie Brilliant Blue or by the silver staining procedure of Oakley et al. (19). Nondenaturing gel electrophoresis on $0.3 \%$ agarose-2.5\% acrylamide gels was performed as described by Liu et al. (20).

Isoelectric focusing/SDS-polyacrylamide gel electrophoresis (SDS-PAGE). Digestion of trypsin-treated samples was terminated by addition of $1.0 \mathrm{mM}$ DFP and the samples were dialyzed against $9.5 \mathrm{M}$ urea, $2 \% \mathrm{NP}-40$, and $5 \% \beta$-mercaptoethanol for $6 \mathrm{~h}$ at $22^{\circ} \mathrm{C}$. Carrier ampholytes $(1.6 \% \mathrm{pH}$ 5-7 and $0.4 \%$ pH 3-10, final concentrations) were added, and the samples were electrofocused for $16 \mathrm{~h}$ at $400 \mathrm{~V}$ in $4 \%$ polyacrylamide tube gels $(0.4 \times 10 \mathrm{~cm})$ containing $1.6 \% \mathrm{pH}$ 4-6 and 0.4\% pH 3-10 carrier ampholytes as described by $O^{\prime}$ Farrell (21). SDS-PAGE in the second dimension was performed on $10 \%$ acrylamide slab gels $(0.3 \times 12 \times 80 \mathrm{~cm})$ as described by Laemmli (18). This slab gel apparatus can accommodate six isoelectric focusing tube gels at one time. Perspective line plots of Coomassie Blue-stained two-dimensional isoelectric focusing/SDS-PAGE were generated using a densitometer equipped with a stage that would step the gel $1 \mathrm{~mm}$ between scans. The total protein in a spot was determined from the summation of the integrated volume elements in successive scans. The gels were oriented so that SDS-PAGE was the scanning dimension and isoelectric focusing was the stepping dimension.

\section{RESULTS}

Reproducibility of tryptic digestion of spectrin from normal volunteers. Digestion of purified spec- trin at $0^{\circ} \mathrm{C}$ with TPCK-trypsin produces a reproducible spectrum of intermediate size peptides with the principle species ranging in molecular mass from 20,000 to 120,000 daltons (Fig. 1). The quantity of material associated with the high-molecular mass bands $(50,000-120,000)$ decreases with a concomitant increase in the staining intensity of the low-molecular mass bands $(22,000-54,000)$ as the enzyme/substrate ratio was stepwise varied from 1:400 to 1:10. An enzyme/substrate ratio of 1:100 was chosen for subsequent investigation of patient spectrin because all of the bands showed good staining intensity and because changes in the 80,000 -dalton peptide reported to be involved in spectrin tetramer formation could be detected (11-13). While the peptide patterns obtained from limited tryptic digestion are extremely consistent for samples extracted, digested, and electrophoresed concurrently, some variations are observed from one experiment to the next. For this reason control normal erythrocytes are always extracted, digested, and electrophoresed along with patient samples. With the exception of a band at 27,000 daltons, identical peptide patterns have been obtained when either crude spectrin extracts or purified spectrin heterodimers are digested at $0^{\circ} \mathrm{C}$ for $20 \mathrm{~h}$. In addition, the peptide pattern was found to be unaffected by conversion of the tetramers to dimers by incubation of the crude spectrin extracts at $37^{\circ} \mathrm{C}$ for $20 \mathrm{~min}$ (Fig. 2).

The peptide patterns produced by limited tryptic digestion of 40 normal volunteers were similar and reproducible (Fig. 1). Although no variations were observed in the spectrin digests from these individuals in the 70,000 - to 110,000 -dalton range, some variability was found in lower molecular mass polypep-

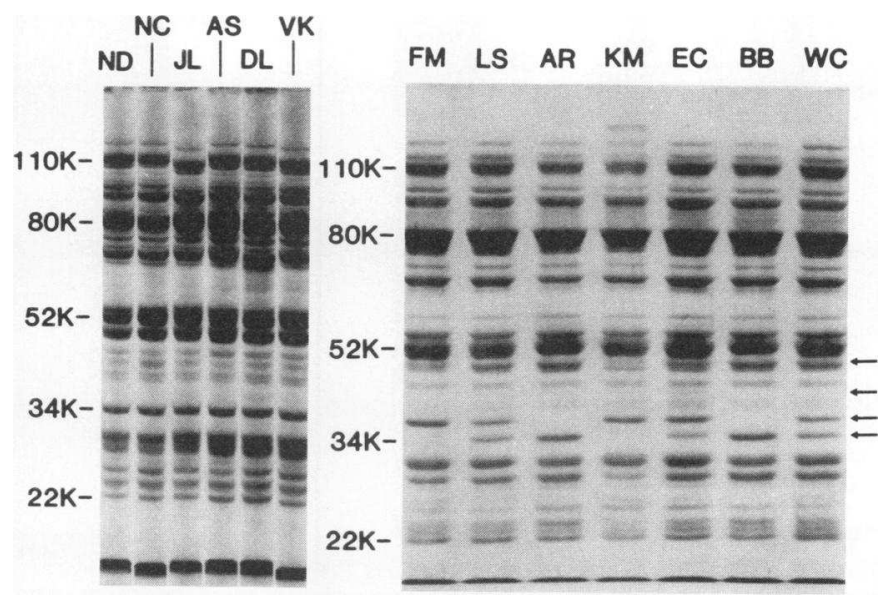

Figure 1 Limited tryptic digestion of spectrin from control normal volunteers. Spectrin extracts from normal volunteers were treated with TPCK-trypsin $(1: 100)$ at $0^{\circ} \mathrm{C}$ for $20 \mathrm{~h} .80$ $\mu \mathrm{g}$ of protein was loaded onto the tube gels (left) and $60 \mu \mathrm{g}$ was applied to the wells of the slab gel (right). The two gel systems were used interchangeably during the course of this study. The molecular mass is indicated on the left and the positions of variable low-molecular mass bands are indicated by the arrows on the right. 


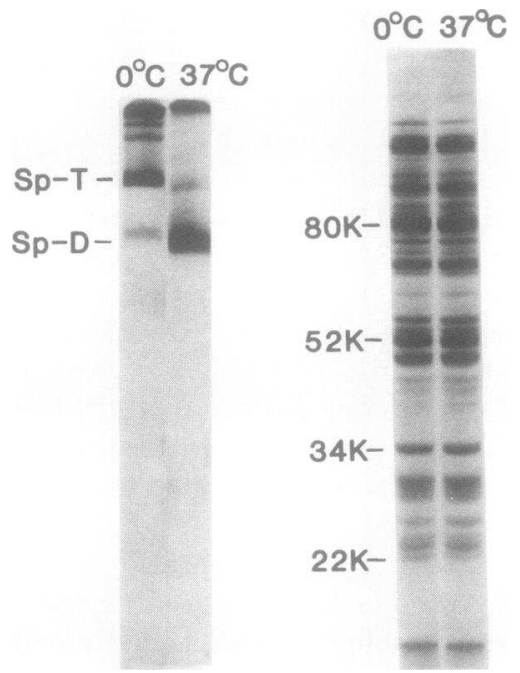

FIGURE 2 Nondenaturing agarose-acrylamide gels (left) and trypsin digests (right) of $0^{\circ} \mathrm{C}$ spectrin extract before $\left(0^{\circ} \mathrm{C}\right)$ and after $\left(37^{\circ} \mathrm{C}\right)$ incubation at $37^{\circ} \mathrm{C}$ for $20 \mathrm{~min}$. The position of the spectrin dimers $(\mathrm{Sp}-\mathrm{D})$ and tetramers $(\mathrm{Sp}-\mathrm{T})$ are indicated.

tides at $34,000,37,000,40,000$, and 47,000 daltons. Some individuals were found to have either the 34,000 dalton polypeptide, the 37,000 -dalton polypeptide, or a variable mixture of both, with the total protein as- sociated with these two bands remaining constant (Fig. 1 , lanes FM, LS, and AR). This variance was observed with a much higher frequency in the normal Black volunteers. A decrease in the staining intensity of the 47,000-dalton polypeptide was found more frequently in individuals who had the 37,000 -dalton polypeptide (Fig. 1, lanes FM and KM). The 40,000-dalton polypeptide is an extremely minor component of the digests as illustrated in Fig. 1; it is present in lane EC and absent in lane BB.

Tryptic digestion of HPP patient spectrin. Limited tryptic digestion of the three HPP patients (N.E., A.B., and D.B.) revealed a similar and reproducible decrease in the staining intensity of the major polypeptide at 80,000 daltons with a concomitant increase in a 74,000-dalton polypeptide as compared with controls (Fig. 3). Identical results were obtained each time these individuals were studied over a 12 -mo period. Densitometer scans of Coomassie Blue-stained gels loaded with an appropriate quantity of protein to give linear color yield for the 80,000 -dalton fragment $(\sim 40-50 \mu \mathrm{g}$ total protein), indicated that it was reduced by $39 \pm 7 \%$ (mean \pm SD for six determinations, two each for N.E., A.B., and D.B.) as compared with controls (22). However, two-dimensional isoelectric focusing/SDS-PAGE indicates that densitometer scans

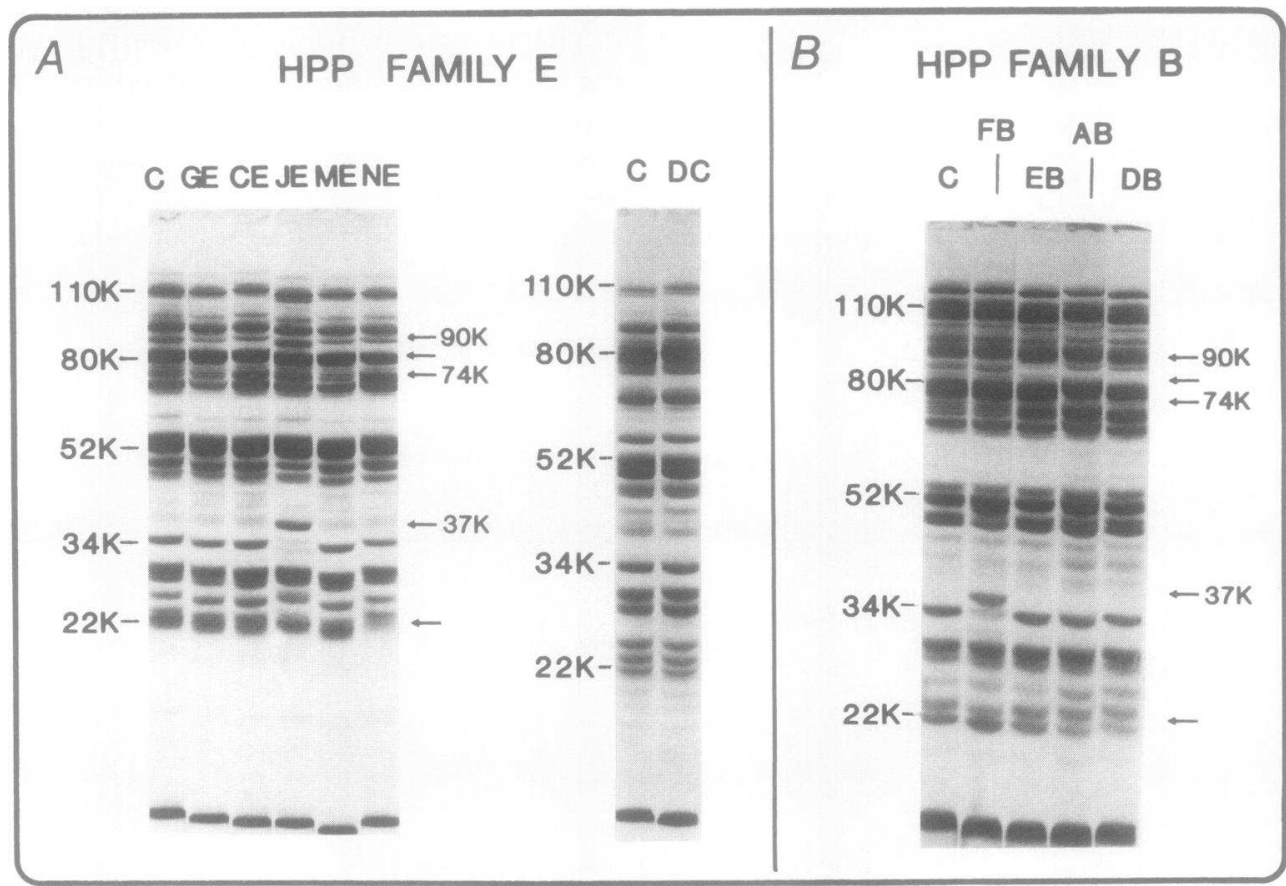

FIGURE 3 Limited tryptic digestion of HPP spectrin. (A) Spectrin extracts from HPP patient N.E., her sister (M.E.), half-brother (J.E.), mother (C.E.), grandmother (G.E.), father (D.C.), and control (C) normal volunteers were prepared, digested, and electrophoresed concurrently. The positions of variable polypeptides are indicated by the arrows on the right. (B) Spectrin extracts from HPP patients A.B. and D.B., their mother (E.B.), their father (F.B.), and a control (C) normal volunteer were prepared, digested, and electrophoresed concurrently. 
of one-dimensional separations underestimate the magnitude of the decrease in the affected 80,000-dalton fragments (Fig. 7). In addition to the changes in these principal polypeptides, a decrease in the staining intensity of minor bands at 88,000 and 22,000 daltons with a concomitant increase in a 90,000 -dalton polypeptide was also observed in tryptic digests of HPP patient spectrin (Figs. 4 and 5).
Tryptic digestion of spectrin from other family members. The asymptomatic mothers (C.E. and E.B.) of the probands, which we have previously found to have partially defective association of spectrin dimers to tetramers (8), displayed a similar diminution of the 80,000 - and 22,000-dalton polypeptides and increase in the 74,000-dalton polypeptide as their affected HPP kindred, but to a lesser extent (Figs. 3-5). In addition,

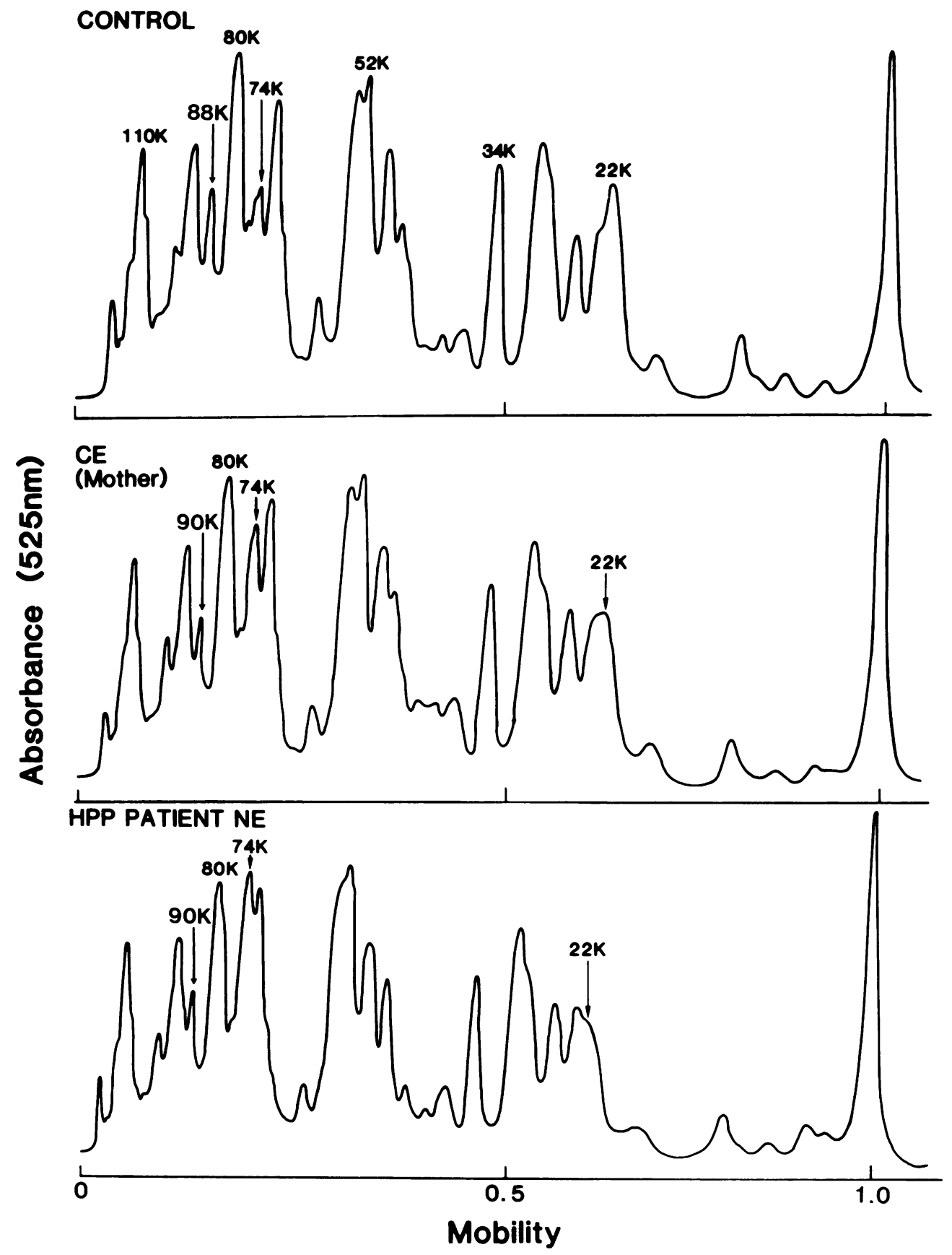

Figure 4 Densitometer scans of the gels in Fig. 5A of trypsin digests of spectrin extracts from HPP patient N.E., her mother (C.E.), and the control normal volunteer. 

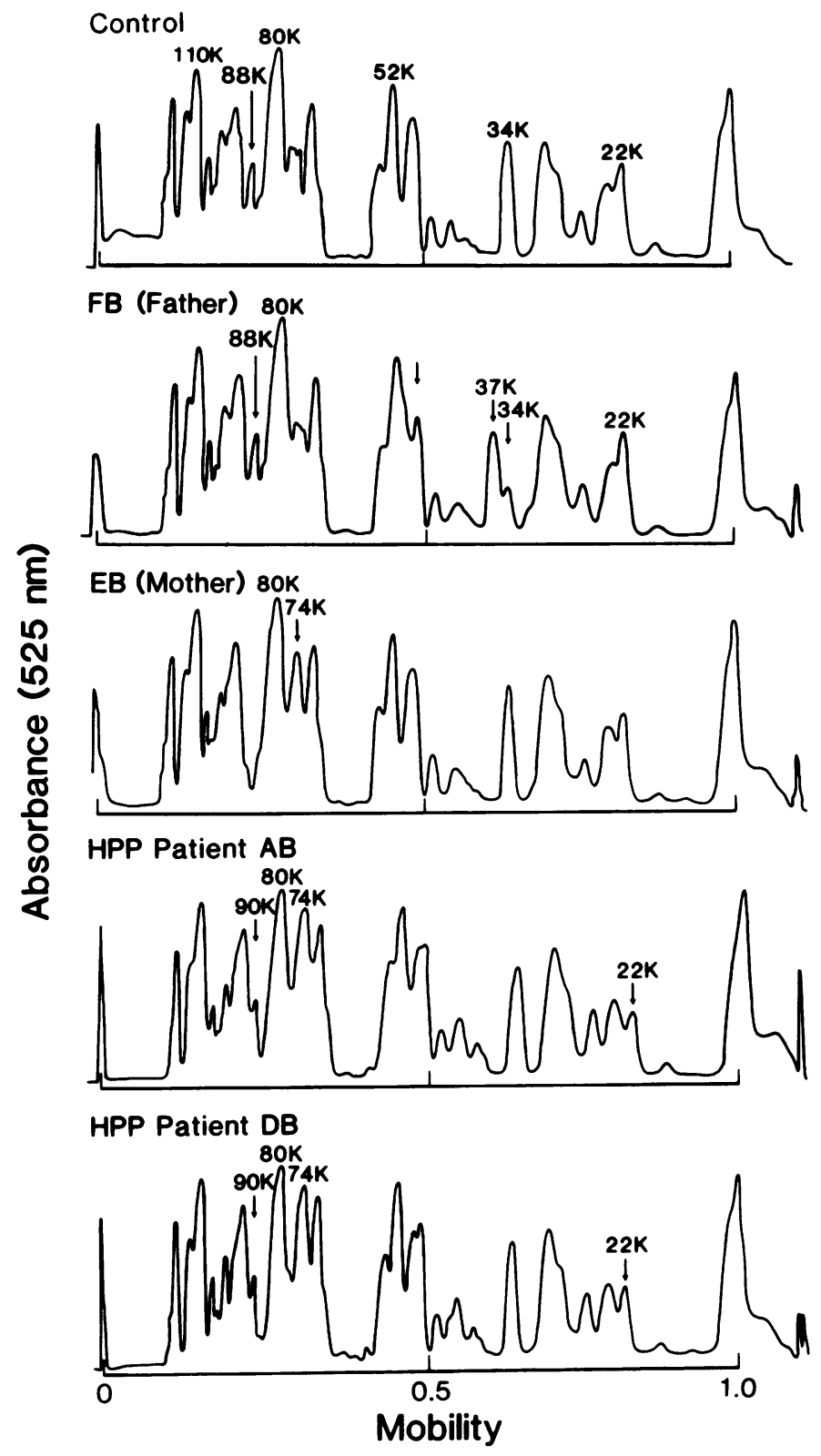

Figure 5 Densitometer scans of the gels in Fig. 5B of trypsin digests of spectrin extracts from HPP patients A.B. and D.B. and their mother (E.B.), father (F.B.), and the control normal volunteer.

one of the proband's mother (E.B.) lacked both the 88,000- and 90,000-dalton polypeptides, while the other proband's mother (C.E.), sister (M.E.), and grandmother (G.E.) all had decreased 88,000- and increased 90,000 -dalton polypeptides. The latter two individuals (M.E. and G.E.) have a normal amount of spectrin dimer in their $0^{\circ} \mathrm{C}$ extracts $(5 \pm 2 \%)$ and an otherwise normal tryptic peptide pattern. Thus, only changes in the $80,000-, 74,000-$, and 22,000 -dalton polypeptides could be correlated to defective spectrin self-association. The father (F.B.) of two of the probands (A.B. and D.B.) and the half-brother (J.E.) of the other had a decrease in the 34,000 - and 47,000 dalton polypeptides and increase in the 37,000 -dalton polypeptides, which was observed in a subpopulation of the control normal volunteers. The half-brother (J.E.) also had a small but reproducible increase in the quantity of 74,000-dalton polypeptides. The other proband's father (D.C.) also produced a peptide pattern indistinguishable from normal volunteers. Both fathers 
(F.B. and D.C.) were hematologically normal, had normal amounts of spectrin dimers (8), and normal skeletal stability. The probability of fatherhood for F.B. for the probands A.B. and D.B. was $95.1 \%$ based on HLA typing $(23,24)$. The paternity testing for D.C. revealed a probability of $>99 \%$ based on HLA typing and hemoglobin electrophoresis $(23,24)$.

Two-dimensional isoelectric focusing/SDS-PAGE. Two-dimensional isoelectric focusing/SDS-PAGE resulted in a reproducible separation of the major tryptic fragments over a range between $\mathrm{pH} 5.0$ and 6.0 (Fig. $6)$. Although some variations in the minor peptides were observed in the normal volunteers, the principal fragments were reproducible and will be described here. The 80,000-dalton polypeptides consistently focused to multiple spots ranging in isoelectric point from $\mathrm{pH} 5.3$ to 5.4 with an additional spot at $\mathrm{pH} 5.65$ (Figs. 6-8). Two-dimensional analysis of spectrin extracts from the HPP patients showed a diminution of the 80,000-dalton spots with an increase in the staining intensity of spots at 74,000 daltons similar to that observed in one dimension (Figs. 6-8). Only the 80,000 dalton spots that focused to $\mathrm{pH} 5.3$ to 5.4 were affected and the 74,000-dalton polypeptides also focused to multiple spots in this range. Densitometer scans indicate that the amount of protein associated with the 80,000-dalton spots between pH 5.3 and 5.4 was decreased by $32-48 \%$ in the HPP patients as compared with controls loaded with comparable amounts of total protein (Fig. 7). The two related probands (A.B. and D.B.) also showed a small but reproducible increase in molecular mass and shift towards higher $\mathrm{pH}$ of two spots at 48,000 daltons (Fig. 8). A spot at 34,000 daltons displayed a comparable shift towards higher $\mathrm{pH}$ (Fig. 8 ). In the case of the two adjacent $48,000-50,000$-dalton spots we cannot determine if both spots shifted in tandem or if one spot "hopped" over the other. These variations were not observed in N.E. or her kindred.

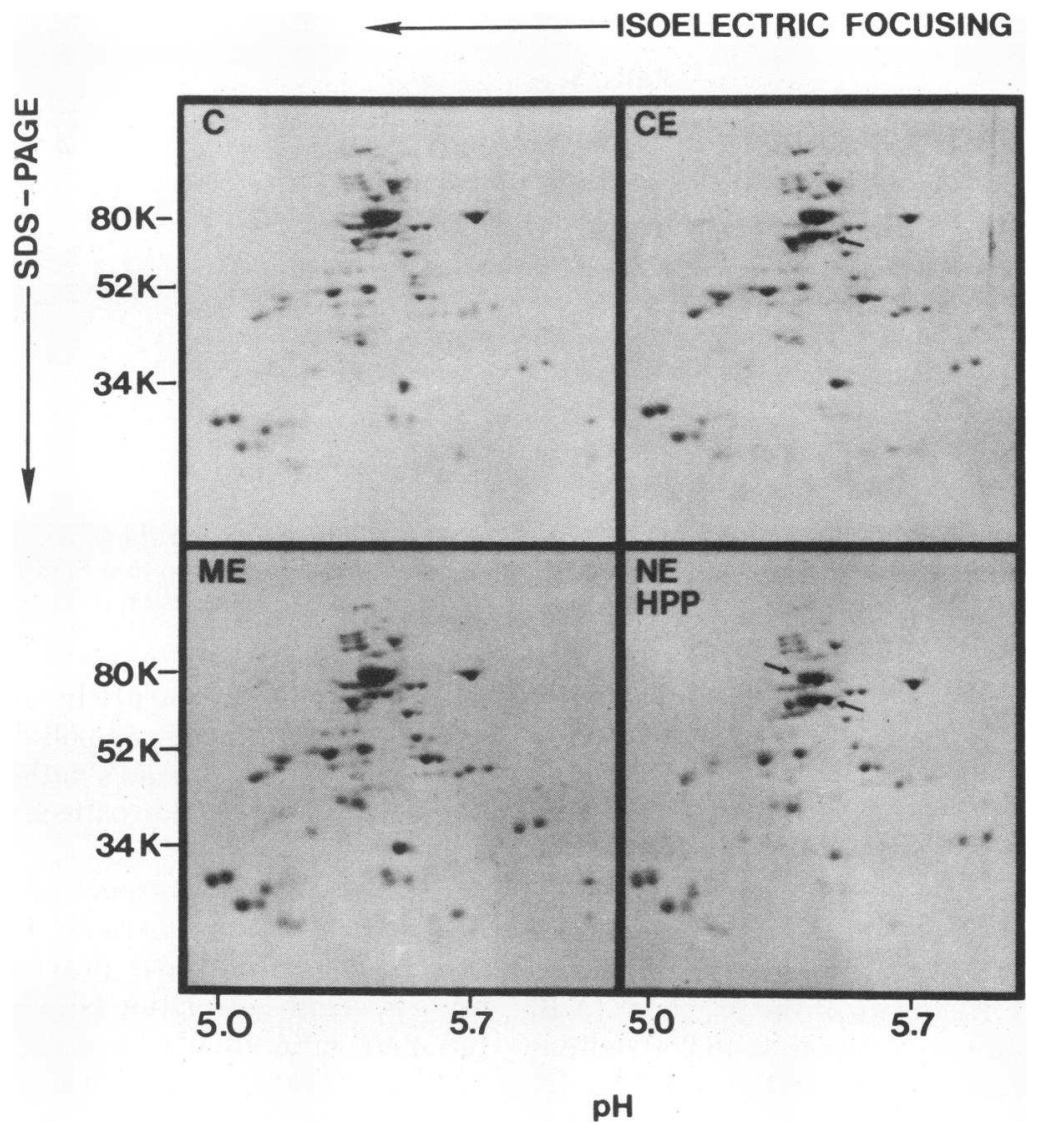

FIGURE 6 Two-dimensional SDS-PAGE/isoelectric focusing of tryptic digests of HPP spectrin. Tryptic digest of spectrin extracts from HPP patient N.E., her sister (M.E.), her mother (C.E.), and a control (C) normal volunteer were electrofocused on pH 4 to 6 gradients as described by O'Farrell (21). The focusing gels were equilibrated with Laemmli (18) sample buffer and electrophoresed concurrently on a single $10 \%$ slab gel. The arrows indicate position of the affected 80,000 - and 74,000-dalton spots. 


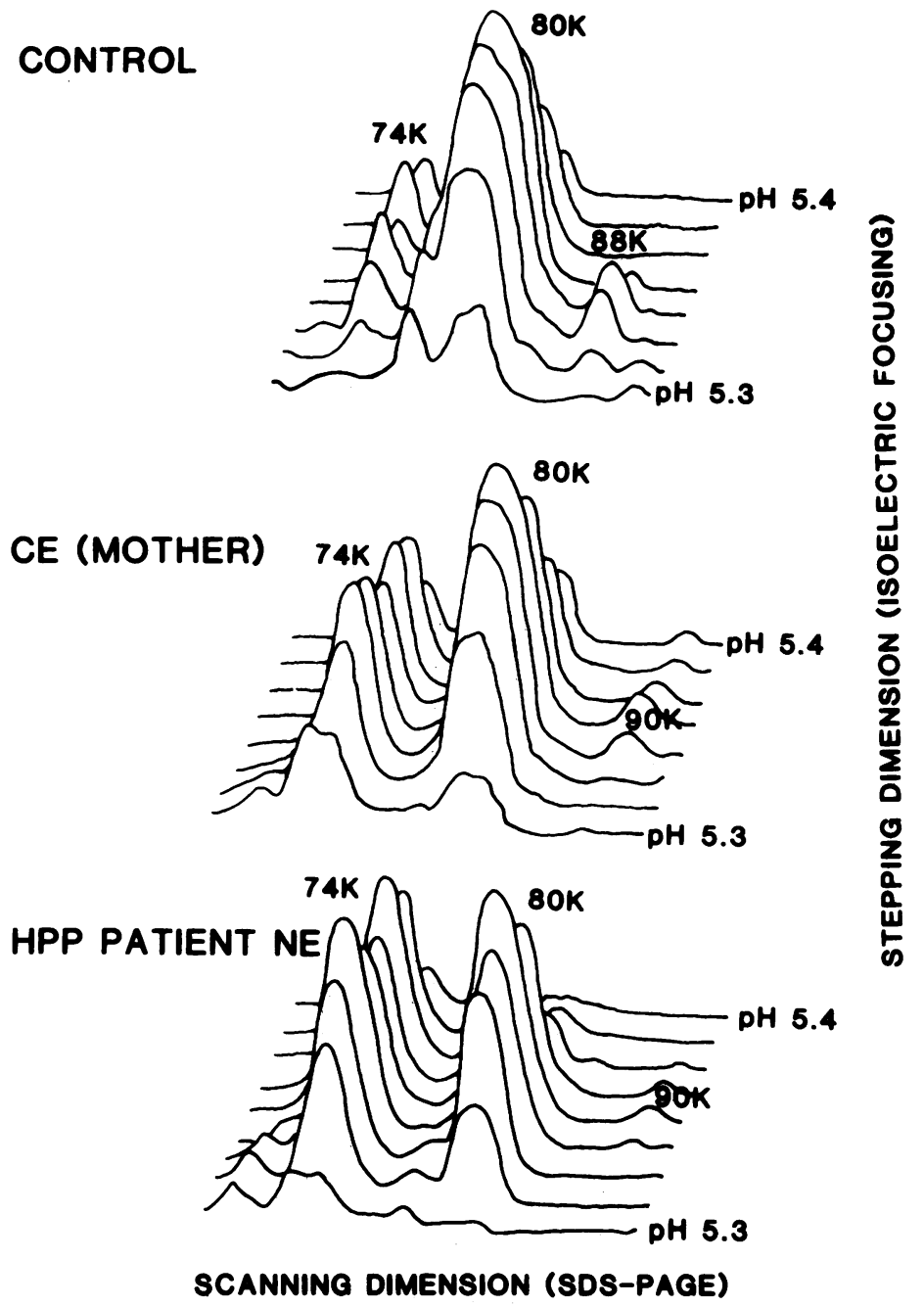

FIGURE 7 Perspective line plots of 80,000 - and 74,000-dalton spots from the two-dimensional isoelectric focusing/SDS-PAGE shown in Fig. 6. The gels were oriented so that SDS-PAGE was the scanning dimension and isoelectric focusing was the stepping dimension.

The mothers of the probands had an increase in the amount of protein associated with the 74,000-dalton spots and decrease in the 80,000-dalton spots, which was again intermediate in magnitude between the HPP patients and controls (Figs. 6-8). Densitometer scans indicate that the amount of protein associated with the 80,000-dalton spots between $\mathrm{pH} 5.3$ and 5.4 was decreased by $20-27 \%$ as compared with controls (Fig. 7). The father (F.B.) of two of the probands (A.B. and D.B.) showed a shift in the spots at 48,000 daltons comparable to that of his progeny (Fig. 8). This individual (F.B.) also has an increase in the 37,000-dalton and decrease in the 34,000-dalton polypeptides, which was frequently observed in normal control volunteers. In two-dimensional isoelectric focusing/SDSPAGE the 37,000- and 34,000-dalton polypeptides focus to the same isoelectric point. In F.B. they are both shifted towards higher $\mathrm{pH}$ by an amount comparable to that observed for the 34,000-dalton spot in A.B. and D.B. The other proband's father (D.C.) produced a two-dimensional peptide pattern indistinguishable from controls.

Evidence for the presence of two forms of spectrin in HPP patients and carriers. Comparison of the data presented here with our previous data indicates that there is a close correlation between the spectrin dimertetramer ratio in $0^{\circ} \mathrm{C}$ extracts and the amount of $80,000-, 74,000-$, and 22,000 -dalton polypeptides in trypsin digestion (8). To further examine this relationship we separated the spectrin dimers and tetramers by sedimentation on linear $5-20 \%$ sucrose gradients and subjected them to trypsin digestion. The spectrin in $0^{\circ} \mathrm{C}$ extracts was found to partition equally into dimer and tetramer for two of the probands (A.B. 


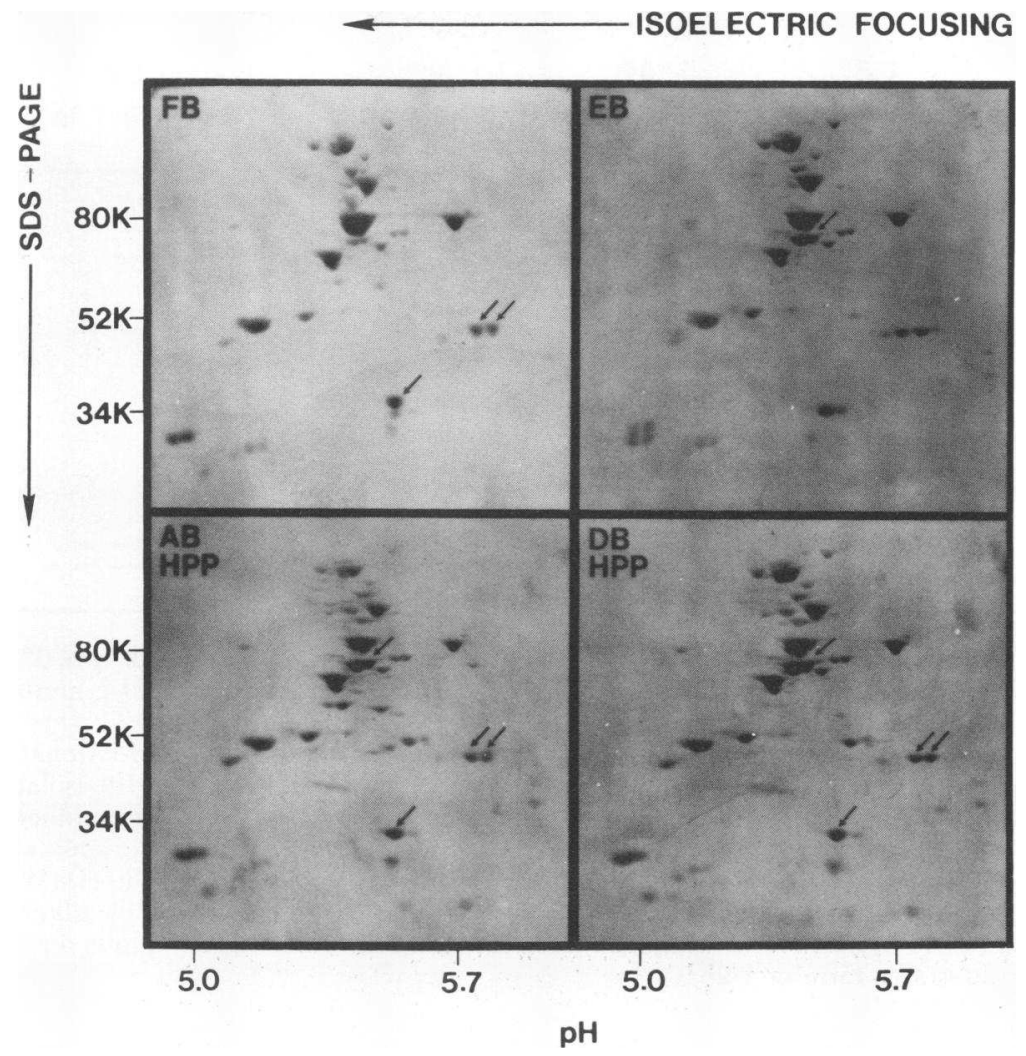

FIGUR: 8 Two-dimensional SDS-PAGE/isoelectric focusing of the tryptic digests of the family B kindred. Tryptic digests of spectrin extracts from HPP patients A.B. and D.B., their mother (E.B.) and father (F.B.), were electrofocused concurrently on pH 4-6 gradients as described by $\mathrm{O}^{\prime}$ Farrell (21). The arrows indicate the position of the affected 80,000-, 74,000-, 48,000-, $37,000-$, and 34,000-dalton spots.

and D.B.) while the third (N.E.) had approximately twice as much dimer as tetramer (data not shown). An increased quantity of 74,000-dalton polypeptide and a decreased quantity of 80,000 - and 22,000-dalton polypeptides was observed in trypsin digests of spectrin dimers as compared with tetramers (Fig. 9). This effect was much more pronounced in extracts from A.B. as compared with N.E. It is likely that the tetramer peak from patient N.E. is contaminated with spectrin dimers because there is approximately twice as much dimer as tetramer in the $0^{\circ} \mathrm{C}$ extracts and the peaks therefore do not separate well. The peptide patterns presented in Fig. 9 are different from those in Figs. 3 and 5 because the total protein concentration was approximately five times lower in these samples (the enzyme to substrate ratio is still $1: 100$ ) and the protein bands have been visualized by silver staining. Similar results were obtained when $0^{\circ} \mathrm{C}$ extract from the mother (E.B.) of two of the patients was fractionated by gel filtration. The spectrin dimers produced a greater increase in the 74,000-dalton polypeptide and decrease in the 80,000 - and 22,000-dalton polypeptides as compared with both spectrin tetramers and high-molecular weight complex (Fig. 9). The decrease in the 22,000-dalton polypeptide in the dimer peak is more readily visualized when the enzyme to substrate ratio is $1: 25$ (Fig. 9B).

\section{DISCUSSION}

The peptide pattern produced by limited tryptic digestion of spectrin from all three HPP patients in this study showed a comparable decrease in the 80,000 dalton polypeptide and increase in the 74,000-dalton polypeptide (Table I). The 80,000 -dalton polypeptide is equivalent to the functional domain of the $\alpha$-chain involved in spectrin dimer-dimer contract described by Morrow et al. (11) in terms of sensitivity to trypsin, molecular mass, and isoelectric point. The fact that the functional domain involved in spectrin tetramer formation is affected in HPP is consistent with our previous observation of defective dimer association of HPP spectrin $(8,11-13)$. Whether or not the 74,000 dalton polypeptide (which increases concomitant to the decrease in the 80,000 -dalton polypeptide) is derived from the 80,000 -dalton domain is presently not 


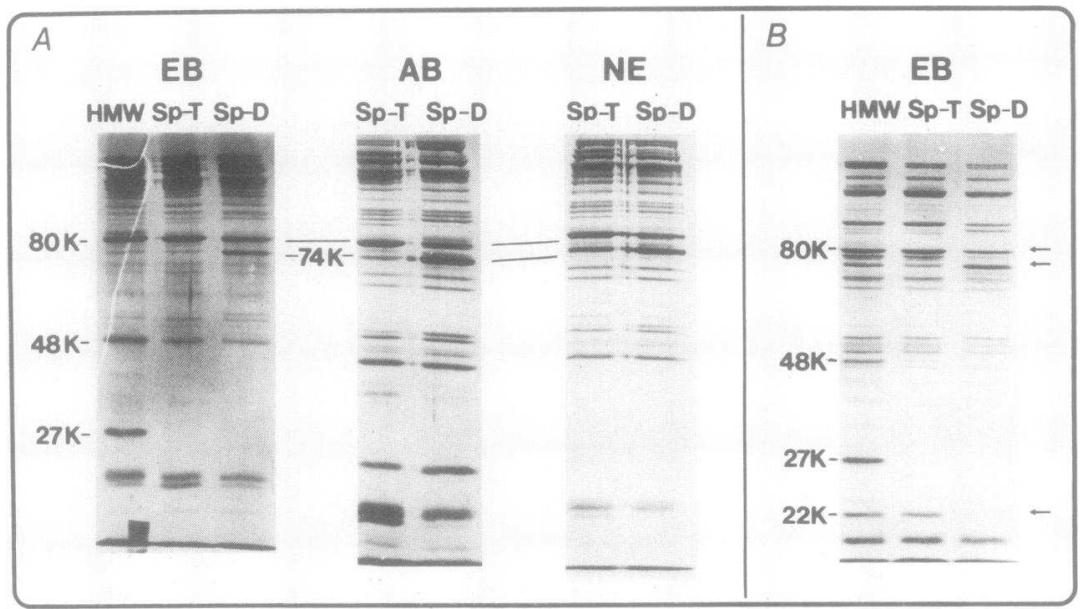

FIGURE 9 Limited tryptic digestion of spectrin dimers and tetramers isolated from $0^{\circ} \mathrm{C}$ extracts of HPP patients A.B. and N.E. and one of the probands (A.B.) asymptomatic mother (E.B.). (A) HPP patient (A.B. and N.E.) spectrin dimers (Sp-D) and tetramers (Sp-T) were separated on linear $5-20 \%$ sucrose gradients while spectrin extract from E.B. was fractionated by gel filtration chromatography on Sepharose 4B. The latter separation permitted the isolation of the high-molecular weight complex (HMW) but required three to four times more blood than the sedimentation procedure. Note that the 27,000-dalton band observed in digest of spectrin extracts but not in digests of purified spectrin is selectively associated with the HMW (Results). $10 \mu \mathrm{g}$ of digest was applied to each lane and the peptides were visualized by silver stain. (B) Isolated $\mathrm{Sp}-\mathrm{D}, \mathrm{Sp}-\mathrm{T}$, and HMW from the mother (E.B.) of one of the probands digested at an enzyme to substrate ratio of 1:25.

certain. This possibility is suggested by the finding that trypsin is capable of removing a 6,000-dalton terminal portion of the 80,000 -dalton polypeptide (13). The resulting 74,000-dalton polypeptide no longer has the ability to bind to native spectrin (13). On the basis of the above data, we propose that HPP spectrin is functionally defective because of a conformational change that affects the 80,000-dalton dimer contact site, rendering it more susceptible to further degradation to 74,000 daltons. Two-dimensional "fingerprinting" is currently underway in our laboratory to establish that the 74,000-dalton polypeptide is in fact derived from the 80,000 -dalton polypeptide.

The decrease in the 80,000-dalton polypeptide and the quantity of 74,000-dalton polypeptide observed in tryptic digests of the HPP patients and the asymptomatic carriers was proportional to the amount of dimer in their $0^{\circ} \mathrm{C}$ extracts (Table I). The differences observed in the tryptic peptide pattern are not due to differences in the proteolysis of spectrin dimer vs. tetramer: as shown in Fig. 2 the same peptide pattern is observed for normal spectrin both in the dimeric and tetrameric forms. As indicated by the tryptic digestion of spectrin dimers and tetramers, which were isolated from $0^{\circ} \mathrm{C}$ extracts of the HPP patients A.B. and N.E. and the asymptomatic carrier E.B. the quantity of 80,000 - and 74,000-dalton polypeptides reflect the percentage of presumed mutant spectrin, which is principally in the dimeric form.
Based on the fact that HPP patients frequently have two clinically normal parents, HPP is considered an autosomal recessive disease; both parents are presumed heterozygous carriers of HPP and their affected progeny would be homozygous. The molecular characterization presented here has some implications regarding the genetics of this disease. The fact that the increase in the 74,000 - and decrease in 80,000 - and 22,000 dalton polypeptides is confined almost exclusively to the spectrin dimer pool, both in the HPP patient A.B. and his asymptomatic mother (E.B.), indicates that these individuals have two pools of spectrin; the normal spectrin molecules are principally tetrameric, while the abnormal spectrin molecules are principally dimeric. Thus, the data presented here does indicate that the asymptomatic mothers (E.B. and C.E:) are heterozygous for HPP and do contain a population of functionally defective spectrin molecules. These individuals can be described as carriers of HPP trait. In the case of the HPP patients, the heterogenity in tryptic peptide pattern for spectrin dimers and tetramers isolated from both A.B. and N.E. in conjunction with the fact that their fathers (F.B. and D.C.) have normal spectrin dimer levels and normal amounts of 80,000 and 74,000-dalton polypeptides indicates that these HPP probands are not homozygotes for the HPP defect (Table I). It is possible that they have inherited a second, as yet unidentified, genetic defect from their fathers and are therefore double heterozygotes. This ad- 
ditional defect may involve another structural or regulatory component of the skeleton or may involve the structure of spectrin in a way that is undetectable by peptide mapping of limited tryptic digests (25). The shift towards higher $\mathrm{pH}$ of spots at $48,000,37,000$, and 34,000 daltons in the two-dimensional peptide patterns of F.B., A.B., and D.B. may be indicative of a second molecular defect. However, since the two-dimensional system provides much more information, a larger population of normal volunteers must be studied so that asymptomatic variants in the normal population may be identified. An alternative hypothesis is that the probands in this study have a defect in the biosynthesis of one of the chains of spectrin in conjunction with the structural defect associated with HPP. A quantitative defect in one, of possibly several, genes coding for one of the spectrin chains (possibly present in the asymptomatic fathers) could be clinically and biochemically silent. According to this hypothesis the affected HPP patients may carry both a quantitative defect (presumably inherited from their asymptomatic fathers) and a structural defect, which they have inherited from their mothers. This hypothesis would explain why the HPP patients have greater amounts of abnormal spectrin than their asymptomatic mothers. It should be further pointed out that the mixture of normal and abnormal spectrin could also result from a posttranslational modification. While dimer to tetramer transformation either in solution (5) or in the membrane (6) is unaffected by spectrin phosphorylation, the effect of methylation, acetylation, or proteolytic damage has not been studied.

The peptide pattern produced by limited tryptic digestion of HPP patient extracts was found to be constant during the course of this study. The probands ranged in age from 1.5 to $14 \mathrm{yr}$ and were from two unrelated families. In addition, the decrease in mechanical stability of triton shells and the increase in the amount of spectrin dimers present in $0^{\circ} \mathrm{C}$ extracts correlated well with the magnitude of changes in the tryptic peptides (Table I). These changes in the tryptic peptide pattern have not been found in any other disease state except for a subpopulation of $\mathrm{HE}$, also characterized by defective spectrin self-association (9). In contrast to HPP, these patients exhibit an autosomal dominant mode of inheritance. The increase in the amount of spectrin dimers in $0^{\circ} \mathrm{C}$ extracts is comparable to that of the asymptomatic HPP carriers. Whether or not the same, or a very similar, genetic defect is involved is uncertain. The possibility that the same genetic defect is involved is supported by the following observations that suggest a link between HPP and some forms of HE: (a) HPP is clinically and morphologically similar to homozygous, mild HE; $(b)$ spectrin and erythrocyte heat stability are decreased in HPP and some forms of HE, particularly during infancy; and $(c)$ both conditions are present in some families $(26,27)$. We are currently using two-dimensional peptide fingerprinting to compare normal, HPP, and $\mathrm{HE}$ spectrins. If the same genetic defect is present in HPP and some forms of HE, then it may be that other, as yet unidentified, factors can affect erythrocyte morphology and determine the clinical presentation. We are also studying patients that make the transition from HPP to HE in their infancy, in an effort to further elucidate these questions.

\section{ACKNOWLEDGMENTS}

We are grateful to the E. and B. families for their help and cooperation during the course of this study. Expert technical assistance was provided by Nelson Chu and P. Y. Aileen Liu. The art work was done by Cela Lamoureux and the manuscript was typed by Lucille Paul.

This research was supported by National Institutes of Health grant HL 27215.

\section{REFERENCES}

1. Marchesi, V. T. 1979. Functional proteins of the human red blood cell membrane. Semin. Hematol. 16: 3-20.

2. Lux, S. E. 1979. Spectrin-actin membrane skeleton of normal and abnormal red blood cells. Semin. Hematol. 16: $21-51$

3. Branton, D., C. M. Cohen, and J. Tyler. 1981. Interaction of cytoskeletal proteins on the human erythrocyte membrane. Cell. 24: 24-32.

4. Ji, T. H., D. J. Kiehm, and L. R. Middaugh. 1980. Presence of spectrin tetramers on the erythrocyte membrane. J. Biol. Chem. 255: 2990-2993.

5. Ungewickell, E., and W. Gratzer. 1978. Self-association of human spectrin. A thermodynamic and kinetic study. Eur. J. Biochem. 88: 379-385.

6. Liu, S. C., and J. Palek. 1980. Spectrin tetramer-dimer equilibrium and the stability of erythrocyte membrane skeletons. Nature (Lond.). 285: 586-588.

7. Zarkowsky, H. S., N. Mohandes, C. S. Speaker, and S. B. Shohet. 1975. A congenital hemolytic anemia with thermal sensitivity of the erythrocyte membrane. $\mathrm{Br} . \mathrm{J}$. Haematol. 29: 537-545.

8. Liu, S. C., J. Palek, J. Prchal, and R. P. Castleberry. 1981. Altered spectrin dimer-dimer association and instability of erythrocyte membrane skeletons in hereditary pyropoikilocytosis. J. Clin. Invest. 68: 597-605.

9. Liu, S. C., J. Lawler, J. Prchal, and J. Palek. 1981. Defective dimer-dimer association and altered tryptic digestion of spectrin in variants of hereditary elliptocytosis. Blood. 58(Suppl. 1): 45a.

10. Speicher, D. W., J. S. Morrow, W. J. Knowles, and V. T. Marchesi. 1980. Identification of proteolytically resistant domains of human erythrocyte spectrin. Proc. Natl. Acad. Sci. USA. 77: 5673-5677.

11. Morrow, J. S., D. W. Speicher, W. J. Knowles, C. J. Hsu, and V. T. Marchesi. 1980. Identification of functional domains of human erythrocyte spectrin. Proc. Natl. Acad. Sci. USA. 77: 6592-6596.

12. Hanspal, M., and G. B. Rolston. 1980. Binding of an 80,000 -dalton trypsin fragment of spectrin to intact spectrin. Proc. Aust. Biochem. Soc. 13: 12.

13. Morrow, J. S., and V. T. Marchesi. 1981. Self-assembly of spectrin oligomers in vitro. A basis for a dynamic cytoskeleton. J. Cell Biol. 88: 463-468. 
14. Coetzer, T., and S. S. Zail. 1981. Tryptic digestion of spectrin in variants of hereditary elliptocytosis. J. Clin. Invest. 67: 1241-1248.

15. Thompson, S., and A. H. Maddy. 1981. The molecular basis of the defect in phosphorylation of spectrin in human hereditary spherocytosis. Biochim. Biophys. Acta. 649: 38-44.

16. Palek, J., S. C. Liu, P. Y. Liu, J. Prchal, and R. P. Castleberry. 1981. Altered assembly of spectrin in red cell membranes in hereditary pyropoikilocytosis. Blood. 57: 130-139.

17. Dodge, J. T., C. Mitchell, and D. J. Hanahan. 1963. The preparation and chemical characteristics of hemoglobinfree ghosts of human erythrocyte. Arch. Biochem. Biophys. 700: 119-130.

18. Laemmli, U. K. 1970. Cleavage of structural proteins during the assembly of the head of bacteriophage T4. Nature (Lond.). 227: 680-685.

19. Oakley, B. R., D. R. Kirsch, and N. R. Morris. 1980. A simplified ultrasensitive silver stain for detecting proteins in polyacrylamide gel. Anal. Biochem. 105: 361363.

20. Liu, S. C., G. Fairbanks, and J. Palek. 1977. Spontaneous, reversible protein cross-linking in the human erythro- cyte membrane. Temperature and $\mathrm{pH}$ dependence. Biochemistry. 16: 4066-4074.

21. O'Farrell, P. 1975. High resolution two-dimensional electrophoresis of proteins. J. Biol. Chem. 250: 40074021.

22. Fazekas de St. Groth, S., R. G. Webster, and A. Datyner. 1963. Two new staining procedures for quantitative estimation of proteins. Biochim. Biophys. Acta. 71: 377392.

23. Bodmer, W. F. 1977. Histocompatibility testing. Munksgaard International Booksellers \& Publishers Ltd., Copenhagen.

24. Walker, R. H. 1978. Paternity testing. Published by the American Association of Blood Banks. 69-136.

25. Agre, P., E. P. Orringer, D. H. K. Chui, and V. Bennett. 1981. A molecular defect in two families with hemolytic poikilocytic anemia. J. Clin. Invest. 68: 1566-1576.

26. Zarkowsky, H. S. 1979. Heat-induced erythrocyte fragmentation in neonatal elliptocytosis. Brit. J. Haematol. 41: 515-518.

27. Lux, S. E., and B. E. Glader. 1981. Disorders of the red cell membrane. In Hematology of Infancy and Childhood. D. G. Nathan and F. A. Oski, editors. W. B. Saunders Company, Philadelphia. 2nd edition. 1: 456-565. 